\title{
PROTEINURIA, OEDEMA, MURMUR AND SKIN RASH - AN INTERESTING CASE OF GUT CARCINOIDS.
}

Khwaja Nizamuddin ST3, Sajjad Ahmad ST5, Raj Thaman Consultant Cardiologist

\section{Case Report:}

A 52 years old man seen in the renal clinic for persistent proteinuria and leg swelling. He also had weight loss, diarrhea for 6 months and getting SOB. Initial impression was Nephrotic syndrome.

Background: Hypertension and chronic depression.

Drug Hx: Olanzapine, Venlafaxine, Ramipril.

Examination: Erythematous rash over the face, limbs and abdomen exacerbated by stress intermittently.

Palpable liver of 3-4 cm with lower limbs and peri-orbital swelling. Investigations: Abdominal US showed grossly enlarged and irregular liver containing multiple lesions of varying echotextures. It was confirmed by CT which also showed a well defined lesion in the mesentery. Liver biopsy confirmed neuroendocrine tumor showing strong positive staining for CD56, chromogranin, NSE and synaptophysin.Ki67 was 3\%. Echo showed signs of right sided heart involvement with severe tricuspid regurgitation and pulmonary stenosis. Interestingly repeat Echocardiogram after a week showed a new onset aortic regurgitation and so confirmed left sided involvement. 24 hours Urinary 5-HIAA was significantly raised at 1384 micromole. He was referred to neuroendocrine MDT.

\section{Discussion:}

Carcinoids are slow growing neuroendocrine tumours originating from Enterochromaffin cells. Incidence is 1.2-2.1 per 100,000 per annum. Metastasis to the liver manifest as carcinoids syndrome and typically cause secretory diarrhea, cutaneous flushing, wheeze and right sided cardiac involvement.

Apart from the typical characteristics of carcinoids syndrome our case highlighted some unusual features like possible membranous Glomerulonephritis (proteinuria) which is associated with solid tumours including carcinoids and severe right sided cardiac involvement extending to the left side of the heart possibly due to excessive amine load.

Left sided cardiac involvement have been previously noted in patients with patent foramen ovale or carcinoid tumour involving the lungs.
Images(with patients consent)
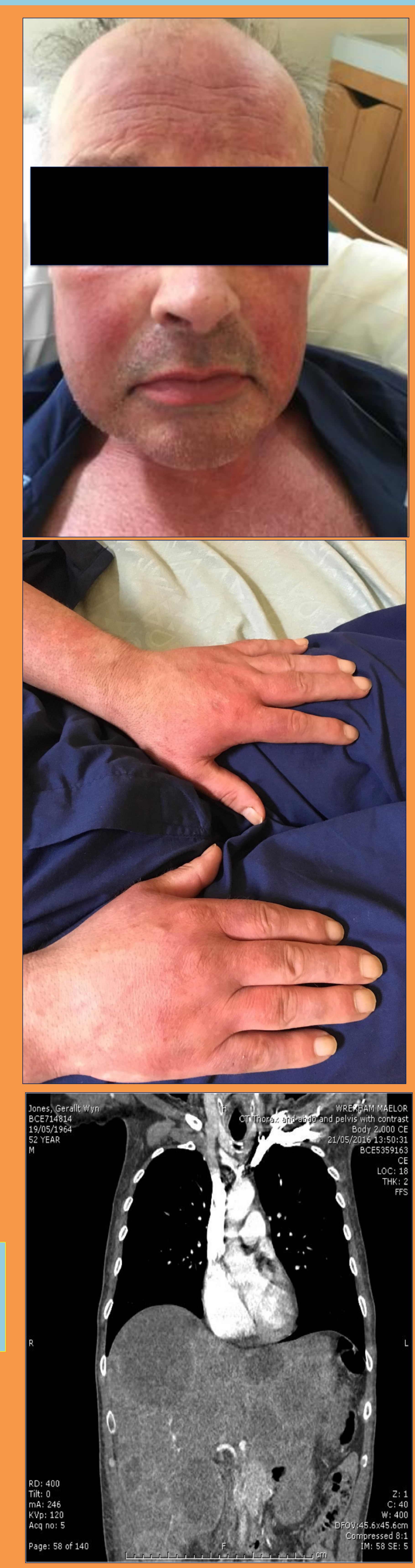

Frequency $(\%) \quad$ Putative mediator

85

Skin

Flushing

Kinins, histamine, kallikreins,other

25

Telangiectasia

Cyanosis

18

Pellagra

7

Excess tryptophan metabolism

\begin{tabular}{|llcl} 
Gl Tract & $\begin{array}{l}\text { Diarrhoea and } \\
\text { cramping }\end{array}$ & $75-85$ & Serotoinin \\
Heart & Right heart & 40 & serotonin \\
\hline & Left heart & 13 & \\
& & &
\end{tabular}

Resp

Wheeze

19

unknown 